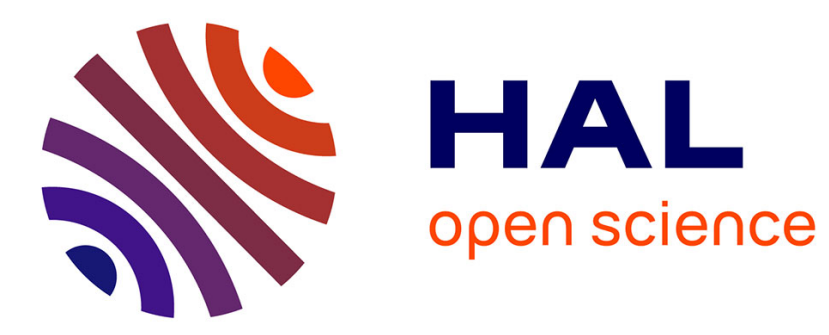

\title{
Analytical model for the prediction of pulsations in a cold-gas scale-model of a Solid Rocket Motor
} \author{
Lionel Hirschberg, Thierry Schuller, Jean Collinet, Christophe Schram,
} Avraham Hirschberg

\section{- To cite this version:}

Lionel Hirschberg, Thierry Schuller, Jean Collinet, Christophe Schram, Avraham Hirschberg. Analytical model for the prediction of pulsations in a cold-gas scale-model of a Solid Rocket Motor. Journal of Sound and Vibration, 2018, vol. 419, pp. 452-458. 10.1016/j.jsv.2018.01.025 . hal-01737077

\section{HAL Id: hal-01737077 \\ https://hal.science/hal-01737077}

Submitted on 19 Mar 2018

HAL is a multi-disciplinary open access archive for the deposit and dissemination of scientific research documents, whether they are published or not. The documents may come from teaching and research institutions in France or abroad, or from public or private research centers.
L'archive ouverte pluridisciplinaire HAL, est destinée au dépôt et à la diffusion de documents scientifiques de niveau recherche, publiés ou non, émanant des établissements d'enseignement et de recherche français ou étrangers, des laboratoires publics ou privés. 


\section{OATAO \\ Open Archive Toulouse Archive Ouverte}

\section{Open Archive TOULOUSE Archive Ouverte (OATAO)}

OATAO is an open access repository that collects the work of Toulouse researchers and makes it freely available over the web where possible.

This is an author-deposited version published in : http://oatao.univ-toulouse.fr/ Eprints ID : 19549

To link to this article : DOI:10.1016/j.jsv.2018.01.025

URL : https://doi.org/10.1016/j.jsv.2018.01.025

To cite this version : Hirschberg, Lionel and Schuller, Thierry Collinet, Jean and Schram, Christophe and Hirschberg, Avraham Analytical model for the prediction of pulsations in a cold-gas scalemodel of a Solid Rocket Motor. (2018) Journal of Sound and Vibration, vol. 419. pp. 452-458. ISSN 0022-460X

Any correspondence concerning this service should be sent to the repository administrator: staff-oatao@,listes-diff.inp-toulouse.fr 


\title{
Analytical model for the prediction of pulsations in a cold-gas scale-model of a Solid Rocket Motor
}

\author{
L. Hirschberg a,b,c, ${ }^{,}$, T. Schuller a,d ${ }^{\text {, J. Collinet }}{ }^{\mathrm{c}}$, C. Schram ${ }^{\mathrm{e}}$, A. Hirschberg ${ }^{\mathrm{f}}$ \\ a Laboratoire EM2C, CNRS et CentraleSupélec, 3 Rue Joliot Curie, 91190, Gif-sur-Yvette, France \\ b Environmental and Applied Fluid Dynamics Dept, von Karman Institute for Fluid Dynamics, 72 Chaussée de Waterloo, 1640, Rhode-St-Genèse, Belgium \\ ${ }^{\mathrm{c}}$ ArianeGroup, 51-61 Route de Verneuil, BP 3002, 78133, Les Mureaux Cedex, France \\ d Université Paul Sabatier, IMFT, CNRS, 2 alle du Professeur Camille Soula, 31400, Toulouse, France \\ e Aeronautics and Aerospace Dept / Environmental and Applied Fluid Dynamics Dept, von Karman Institute for Fluid Dynamics, 72 Chaussée de \\ Waterloo, 1640, Rhode-St-Genèse, Belgium \\ ${ }^{\mathrm{f}}$ University of Twente, Faculty of Engineering Technology, Engineering Fluid Dynamics, P.O. Box 217, 7500, AE Enschede, The Netherlands
}

Keywords:

Vortex sound

Self-sustained pulsations

Solid Rocket Motor

\begin{abstract}
A B S T R A C T
Cold gas scale model experiments (1/30) demonstrate that coupling of vortex shedding with acoustic standing waves can produce pressure oscillations of the same level as observed in large Solid Rocket Motors. An analytical acoustical energy balance model is proposed in which the system is described as a single mode acoustic resonator and the pulsations are assumed to be purely harmonic. The selected acoustic mode number is an input to the model. Quasisteady linear models are used to describe losses of acoustic energy by vortex shedding at a thermal inhibitor ring, radiation at the nozzle and friction within the porous injection wall used for gas injection. The sound production is predicted by using a 2-D planar point vortex model combined with the Vortex Sound Theory. The model demonstrates that the sound production due to interaction of the vortex with the cavity surrounding the integrated nozzle is dominant, explaining previous results of cold gas and hot-gas scale models. The effect of vortex ingestion by the nozzle is negligible. Aspects of the nozzle geometry, other than the cavity volume, are not critical. The model predicts pressure pulsations within a factor 2 , when the circulation of the vortices is taken one third of the maximum available circulation. This reduction factor of the circulation is assumed to be a consequence of turbulence. The Mach number corresponding to the maximum of pulsation is predicted within $20 \%$ in a range comparable to results obtained by axis-symmetrical numerical flow simulations.
\end{abstract}

\section{Introduction}

Solid Rocket Motors (SRMs) can display strong acoustic oscillations [1-3]. For small engines these are often driven by a coupling between the combustion and acoustic standing waves in the engine [4,5]. In large SRMs the oscillations can be sustained by coupling between vortex shedding and acoustic standing waves [6,7]. A modulation of the combustion rate by the acoustic field may also alter these pulsations, but is not absolutely necessary to sustain pulsations.

Detailed numerical simulations (CFD) of these sustained pressure oscillations in SRMs stil remain challenging and were recently reviewed in Ref. [3]. This makes systematic parametric CFD studies of this phenomenon in SRMs wildly impractical. Thus, there is a need for simplified models to accompany these numerical investigations, providing fast results which can suggest 
if an envisioned numerical run is beneficial and providing a framework for interpretation of simulation results. These could eventually be used for interpolation or extrapolation of experimental or numerical results.

Most of the past analytical research effort focuses on growth of instabilities [8-10] which is described using linear theory and as such is not capable of predicting limit cycle amplitudes. The main results were also derived for combustion related instabilities with limited progress made for instabilities triggered by hydrodynamic interactions [8].

Originally [11] it was believed that vortex shedding at thermal inhibitor rings between segments of the propellant, so called Obstacle Vortex Shedding (OVS), was the only possible cause of vortex shedding. However, Vuillot [6] and Dotson et al. [12] showed that vortices can be formed in the absence of inhibitors. This is due to the intrinsic instability of the so-called Taylor flow generated by the combustion $[13,14]$ and is referred to as Surface Vortex Shedding (SVS). Vortex shedding can also occur at the edge of an abrupt expansion in the combustion chamber cross-section. This is called Angle Vortex Shedding (AVS) and this process is similar to OVS. In this paper OVS is focused on as a mechanism for vortex shedding and a model is developed to understand how OVS vortices interact with the sound field in a SRM.

The experimental results display oscillation bursts in specific Mach number ranges corresponding to a particular acoustic mode and a hydrodynamic mode. At the pulsation maximum the oscillation frequency coincides with the acoustic mode eigenfrequency. Within such a burst the oscillation frequency increases with increasing Mach number as observed in actual SRMs $[12,15,16]$. A famous example of such data is shown in Fig. 1.

Fig. 1 is a reproduction of the high amplitude pulsations detected in Titan 4 SRM. One observes bursts of pulsations around the eigenfrequency of the first acoustic mode corresponding to a standing wave of approximately one half wavelength along the combustion chamber. The Mach number at the nozzle inlet decreases with time as the combustion chamber diameter increases due to combustion while the chocked nozzle throat remains almost constant. Within one burst, the frequency decreases as a function of time, hence it increases with increasing Mach number. This frequency signature is a consequence of the time delay in the convection of vortices between the vortex shedding position in the combustion chamber and the nozzle, where sound is generated. Fig. 1 has motivated investigations of OVS as a strong source of instabilities [15-19] in SRMs.

It is known that vortical structures will produce sound by hydrodynamic interaction with the nozzle, but can also as suggested by Matveev and Culick [20] generate sound by a modulation of the combustion. The focus here is on the hydrodynamic interaction with the nozzle.

Anthoine et al. [17-19] confirmed the importance of vortex nozzle interaction in sound production. In the cold gas experiments [17] the combustion is replaced by injection of air through a porous wall to reproduce the flow at a $1 / 30$ scale in a Ariane 5 SRM. Anthoine [17] found, ratios $p_{\text {rms }} / p$ of the root-mean-square (rms) acoustic pulsation amplitude $p_{\text {rms }}$ to static pressure $p$ of the order of $10^{-3}$. This corresponds to the amplitudes observed in full scale firing test [15] and scale models with combustion $[15,21]$. Anthoine observed the characteristic frequency signatures observed in SRMs [12,15,16], an increasing frequency around the acoustic mode frequency with increasing Mach number around each maximum of pulsation amplitude. This confirms that these signatures can be accounted for by convective delay of vortices.

Anthoine et al. [17,18] proposed an analytical model to estimate the pulsation amplitude by balancing the vortex sound power to radiation losses at the nozzle. In the present paper an improved analytical model is provided based on the Vortex Sound Theory in which the losses are estimated using quasi-steady linear models. This includes radiation at the nozzle, the effect of the vortex shedding at the inhibitor [22] and the sound absorption by the porous wall [23]. The effect of convection in the nozzle radiation model, ignored by Anthoine [17], is also included using the expressions from Marble and Candel [24] and

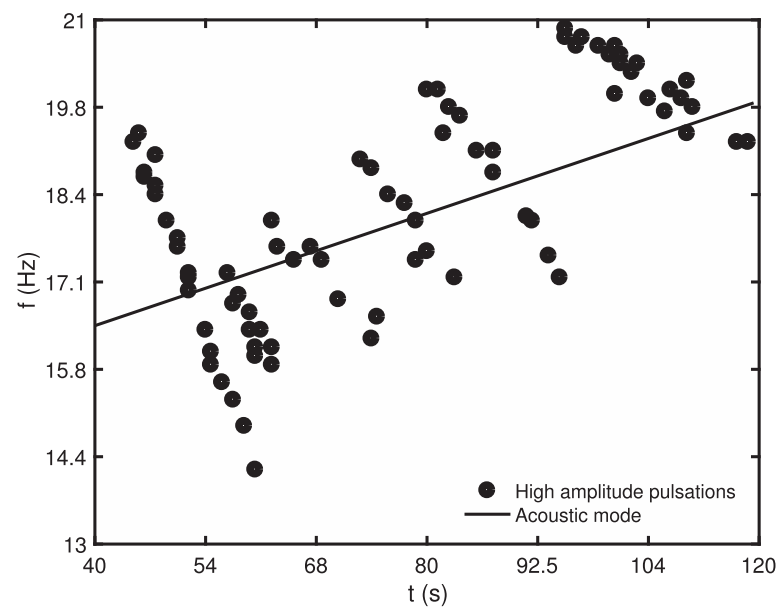

Fig. 1. Time dependence of the frequency of pulsations observed in a SRM. Titan 4 data, Figure after Fig. 4 in Ref. [12]. One observes six sets of measurements corresponding to bursts of high amplitude pulsation. Within each burst the frequency of oscillation decreases with increasing time, corresponding to an increase of oscillation frequency with increasing Mach number. 
Culick [8].

The objective of this paper is to develop an analytical framework that can be used to simulate limit cycles in SRMs focusing on sources of sound due to vortex dynamics only. Another aim is to use the model for parametric investigations of effects due to changes of the nozzle geometry. As a first validation step the proposed model is compared to cold-gas scale model experimental data $[17,25]$.

The first model considered is a nonlinear energy balance, in which saturation of the amplitude is assumed to be reached as a result of the formation of discrete vortical structures. The circulation and path of these vortical structures is assumed to be independent of the acoustical pulsation amplitude, because the acoustically induced velocity perturbations are typically two orders of magnitude smaller than the main flow velocity. Hirschberg et al. [26] developed a model using the same nonlinear energy balance modeling approach. However, the model in Ref. [26], used a crude vortex dynamics model with what amounts to three fit-parameters. The model presented in this paper includes an improved vortex dynamics description that reduces the number of fit parameters to one. It allows for the parametric study of the influence of the shape of the nozzle inlet which was not possible using the model in Ref. [26]. Moreover, the present model takes into account both the contributions of the nozzle cavity volume $V_{c}$ and the ingestion of the vortex by the nozzle as sources of sound, allowing for an analytical investigation of the contribution of these effects that has not been carried out previously.

Additionally to the energy balance, a lumped model is developed. The lumped model allows a prediction of the Mach number dependence of the oscillation frequency $(M / f)(d f / d M)$ due to above mentioned convective effects.

Section 2 describes the cold gas experiments of Anthoine [17] used as a test case for the proposed model. In this section the low amplitude and moderate amplitude regimes are discussed. The model is developed in section 3. Succinct descriptions of the acoustic loss model and of the vortex dynamics model which are used are also made in this section. The mathematical details are given in A and B. Predictions are compared to cold gas experimental data in section 4. Changes of the nozzle shape and the cavity volume $V_{c}$ are also investigated in the section. Conclusions are summarized in section 5.

\section{Cold gas model with axial injection}

Fig. 2 shows a sketch of the axial injection (1/30) scale model of the Ariane 5 booster used by Anthoine [17]. The cylindrical pipe of inner radius $r_{p}$ and length $L_{p}$ is terminated at the upstream end by a porous wall through which air is injected. This air is provided by a reservoir at a pressure $p_{r} \simeq 5 \mathrm{bar}$ and room temperature. The static pressure in the pipe is around $p=$ $p_{r} /(1+19 M)$ [17], where $M$ is the cross-sectional averaged Mach number in the pipe. In the experiments this Mach number varies in the range $0.05 \leq M \leq 0.20$. At the downstream pipe end a submerged (integrated) nozzle is placed. This integrated nozzle has a cavity volume $V_{c}$ representative of the acoustically accessible cavity volume which occurs during full scale firing test. Different nozzle models are used to vary $V_{c}$ (see Table 1 ). The Mach number $M$ in the pipe can be varied by reducing the nozzle throat area by the introduction of a needle. A solid sharp edged orifice of radius $r_{o}<r_{p}$ is placed at a distance $L_{o}$ from the nozzle inlet. This orifice represents a thermal inhibitor emerging from the propellant. It is the vortex generator in the axial injection experiments, which are considered here.

Anthoine [17] considered various models of the cavity corresponding to various stages during a firing test. In the present discussion the focus is on the data for the largest cavities (nozzles 1,7 and 9 in Table 1). Anthoine et al. [18] observed that the pulsation level $p_{\text {rms }} / p$ measured at the upstream head-end wall was proportional to the cavity volume $V_{c}$. This is predicted by the theory, if it is assumed that the sound is generated when the vortices travel along the cavity opening and neglecting the sound generated by the ingestion of the vortices by the nozzle.

A typical result of experiments by Anthoine [17] is shown in Fig. 3 for $L_{p}=0.393 \mathrm{~m}, L_{0}=0.071 \mathrm{~m}, r_{p}=0.038 \mathrm{~m}$ and $r_{0}=$ $0.029 \mathrm{~m}$. In this figure the measured root-mean-square amplitude $p_{\text {rms }}$ of the pressure pulsations measured at the head-end normalized with static pressure $p_{\mathrm{rms}} / p$, is shown as a function of the Mach number $M$. In applications with combustion such as in Ref. [12] the Mach number decreases as a function of time because for a fixed throat diameter the nozzle inlet diameter

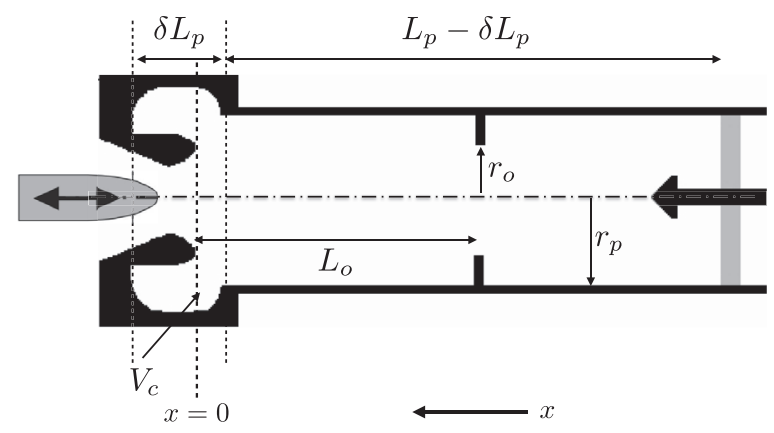

Fig. 2. Axial injection cold gas scale experimental setup. The nozzle inlet is positioned at $x=0$. The positive flow direction is from right to left. Air is injected through the upstream porous head end wall (light grey), the injection is indicated with a big black arrow pointing to the nozzle on the axis of symmetry. The inlet Mach number $M$ is varied by introduction of a needle (dark grey) in the nozzle throat. 
Table 1

Nozzle numbers (column 1 ) and associated cavity volumes $V_{c}$ (column 2) used in cold gas experiments at the VKI [17,25,29]. The scaling factor $\alpha \equiv V_{\text {nozzle } 7} / V_{c}$ is shown in column 3 .

\begin{tabular}{lll}
\hline Nozzle & $V_{c}\left(\mathrm{~m}^{3}\right) \times 10^{-5}$ & $\alpha$ \\
\hline 1 & 20.3 & 0.906 \\
2 & 10.0 & 1.84 \\
5 & 6.71 & 2.74 \\
7 & 18.4 & 1.00 \\
9 & 21.8 & 0.844 \\
\hline
\end{tabular}

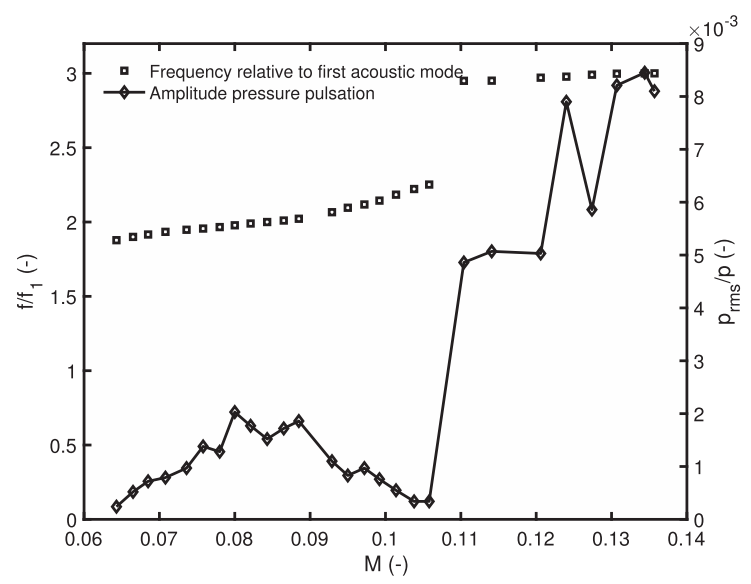

Fig. 3. Pulsation frequency with respect to the acoustic mode frequency (left) and relative rms pressure oscillation level (right) as a function of the Mach number. Figure after Fig. 4.27a in Ref. [17].

increases in time.

The oscillation frequency $f$ is represented in the dimensionless form $f / f_{n}$ in Fig. 3. For each acoustic mode the oscillation frequency increases monotonically with increasing Mach number. The oscillation frequencies are close to the acoustic modes of a closed-closed pipe of length $L_{\text {eff }}$ :

$$
f_{n}=n \frac{c}{2 L_{\mathrm{eff}}}
$$

with $n=1,2,3 \ldots$ where $c$ is the speed of sound in the pipe and

$$
L_{\text {eff }}=L_{p}-\delta L_{p}+\frac{V_{c}}{\pi r_{p}^{2}}
$$

where $V_{c}$ is the nozzle cavity volume and $\delta L_{p}$ is the depth of the nozzle cavity. As a first low frequency approximation the acoustic effect of this cavity is taken into account by increasing the pipe length by $V_{c} /\left(\pi r_{p}^{2}\right)$. The influence of the orifice on the resonance frequency is neglected here. The mean pressure loss across the porous head end wall is large enough to approximate it as a solid wall for the calculation of the resonance frequency. However, the effect of porosity on the acoustic losses is accounted for in the next section. Note that in the acoustic model the head end wall is at $x=-L_{\text {eff }}$.

Due to flow separation at the sharp edges of the orifice a free jet develops downstream of the orifice with a radius $r_{\text {jet }} s$ smaller than the orifice radius $r_{o}$. The ratio $\left(r_{\text {jet }} / r_{o}\right)^{2} \equiv \Upsilon$ is called the vena contracta factor. It is a function of $r_{o} / r_{p}$. Empirical data by Gilbarg [27] is used to determine this vena contracta factor. Here dependence of the vena contracta factor on the Mach number is neglected [28]. For the cases considered by Anthoine [17,29], the pipe radius is $r_{p}=0.038 \mathrm{~m}$ and three different orifice radii are used $r_{o}=0.029 \mathrm{~m}, 0.031 \mathrm{~m}$ and $0.034 \mathrm{~m}$. The vena contracta factors are in these cases $\Upsilon=0.68,0.71,0.78$ respectively.

The fluctuation of the acoustic velocity at the edge of the orifice, induces a modulation of the vorticity in the shear-layer bounding the jet. This triggers a hydrodynamic instability of the shear layer and the formation of coherent vortical structures (vortices). An empirical estimate of the convection velocity $U_{\Gamma}$ of these vortices is $U_{\Gamma}=0.4 U_{\text {jet }}=0.4 U\left(r_{p} / r_{\text {jet }}\right)^{2}[26,30]$ where $U=M c$. Using this estimate, one can estimate the number $m_{h}$ of vortices between the orifice and the nozzle inlet, as:

$$
m_{h}=\frac{f_{n} L_{o}}{U_{\Gamma}}=\frac{\Upsilon}{0.4 M}\left(\frac{n}{2}\right)\left(\frac{L_{o}}{L_{\text {eff }}}\right)\left(\frac{r_{o}}{r_{p}}\right)^{2} .
$$

Maxima in pulsation amplitudes appear to correspond to an integer number of vortices $m_{h}$, which is called a hydrodynamic mode. Each hydrodynamic mode corresponds to a Mach number $M=U / c$ for which the pulsation amplitude reaches a maxi- 
mum and the frequency coincides with an eigenfrequency $f_{n}$ of an acoustic mode $n$ given by Eq. (1). Around the coincidence, the frequency $f$ of the oscillation increases with increasing Mach number as shown by Figs. 1 and 3.

In a previous model [26] the empirical expression of $U_{\Gamma}$ was used and the convection velocity was supposed to remain constant. In the present work a 2-D incompressible planar potential flow model is used to calculate the vortex velocity and path. Assuming a standing wave one can estimate the relative acoustic velocity fluctuations $u_{\text {rms }} / U$ upstream of the orifice using:

$$
\frac{u_{\mathrm{rms}}}{U}=\frac{p_{\mathrm{rms}}}{\gamma p M}\left|\sin \left(k_{n} x_{0}\right)\right|,
$$

where $p_{\text {rms }}$ corresponds to the fluctuations at the pressure anti-node at the upstream head end, and $\gamma=1.4$ is the ratio between the heat capacity at constant pressure and the heat capacity at constant volume i.e. the Poisson constant. In Eq. (4), $k_{n}=2 \pi f_{n} / c$ is the wavenumber and $x_{o}=L_{o}+V_{c} / \pi r_{p}^{2}$ is the effective position of the orifice. The maximum of the observed pressure pulsation level is in the range $10^{-3} \leq p_{\text {rms }} / p \leq 10^{-2}$. Hence this corresponds for $M=\mathcal{O}\left(10^{-1}\right)$ to velocity fluctuations of the order $10^{-2} \leq u_{\mathrm{rms}} / U \leq 10^{-1}$. This corresponds to moderate amplitude levels according to the classification of Bruggeman et al. [30,31]. Further downstream the shear layer breaks down into discrete vortices. One new vortex is formed each time the acoustic velocity in the main flow direction just upstream from the inhibitor passes through a zero amplitude increasing to positive values. This phase condition proposed by Nelson et al. [32] and Bruggeman et al. [30] predicts the moderate amplitude behaviour in many low Mach number self-sustained flow instabilities in cavities and pipe systems.

While the phase of the vortex shedding is controlled by the acoustic field, the magnitude of the circulation of the vortex is independent of the acoustic amplitude. The circulation $\Gamma_{\max }$ corresponding to the amount of vorticity shed during one period of oscillation is an upper bound for the circulation $\Gamma$ of the vortices that reach the nozzle. Once a new vortex is formed at time $t=\tau$, its circulation $\Gamma$ is assumed to grow linearly in time over one period of oscillation $2 \pi / \omega$ until it reaches maturity and its circulation remains constant. The present model assumes for $t-\tau<2 \pi / \omega$ that:

$$
\Gamma=K \frac{\Gamma_{\max } \omega}{2 \pi}(t-\tau)
$$

where

$$
\Gamma_{\max }=0.5 U_{\text {jet }}^{2} \frac{2 \pi}{\omega}
$$

where $U_{\text {jet }}$ is the flow velocity in the jet formed by flow separation at the orifice. $K$ is a constant, supposed to describe the reduction of the circulation of the coherent structures due to turbulent diffusion.

When using this model with $K=1$ assuming that all the vorticity was concentrated into a line vortex, Bruggeman et al. [30] and Tonon et al. [33] found pulsation amplitudes in pipe systems with closed side branches which were about a factor 5 too high. Considering axially symmetric numerical simulations of hot gas scale model experiments Gallier et al. [21] found a reduction of $30 \%$ to $50 \%$ of the predicted pulsation amplitude when turbulence was taken into account with a URANS numerical model compared to laminar flow simulations. Similarly when considering sound production by a compact cavity in a corrugated pipe Golliard et al. [34] also found reduction of acoustic power by a factor 2 when turbulence was taken into account. Hence, one expects that $K$ should be in the range $1 / 5 \leq K \leq 1 / 2$. The parameter $K$ will be fitted to one experiment, and the ability of the model to predict other experiments will be verified.

The linear stability theory for a parallel shear layer flow [35] shows that the growth of linear perturbations of a shear layer is limited to low frequencies such that the hydrodynamic wavelength of the perturbation is about ten times the shear layer momentum thickness. Due to turbulence the shear layer width increases linearly with the distance from the separation point. At low pulsation amplitudes the perturbations may reach the critical shear layer width for which the layer becomes linearly stable before the vorticity has been fully concentrated into a coherent vortical structure. This explains that below a certain pulsation amplitude the vortices have a lower circulation, which is amplitude dependent. The "moderate amplitude" model fails typically for $\left|\mathbf{u}^{\prime}\right| / U<10^{-2}$ where $U=c M$. At these low amplitudes the model tends to overestimate the pulsation level.

In the numerical simulations of Gallier et al. [21] excellent predictions of the pulsation amplitudes are obtained in most of the experiments considered. The only exception is that the model drastically over-predicts or under-predicts the pulsation amplitude in cases for which the observed pulsations were very low. This indicates that in the low amplitude regime the pulsation amplitude is difficult to predict. In experiments one actually observes that the pulsation amplitude in the low amplitude regime can change by orders of magnitude as a result of small modifications in the set-up. At very low amplitudes the circulation $\Gamma$ of the vortices is proportional to the amplitude of the local acoustic velocity fluctuations where the vortices are shed. In that case both losses and sound production depend quadratically on the pulsation amplitude, which implies that the limit cycle amplitude is undetermined.

An overview of the available experimental data is provided in Table 2. One observes that the pulsation amplitude is in most cases in the moderate amplitude regime. Experiments with low amplitudes are indicated by an asterisk. 
Table 2

Overview of the results of experiments, with axial injection obtained with the cold flow scale model of the VKI. In the tables repeating values in columns are replaced by a dashed line.

\begin{tabular}{|c|c|c|c|c|c|c|c|c|}
\hline Num. & Nozzle & $L_{p}(\mathrm{~m})$ & $L_{o}(\mathrm{~m}) \times 10^{-2}$ & $r_{o}(\mathrm{~m}) \times 10^{-2}$ & $\Upsilon$ & $n$ & $m_{h}$ & $\frac{u_{\mathrm{rms}}}{U} \times 10^{-2}$ \\
\hline \multicolumn{9}{|c|}{ (a) Analysis and overview of relevant experimental data from Ref. [17]. } \\
\hline 1 & 7 & 0.393 & 7.1 & 2.9 & 0.68 & 2 & 2.1 & 1.8 \\
\hline 2 & | & 0.393 & | & | & | & 3 & 1.8 & 2.6 \\
\hline 3 & | & 0.305 & 1 & 1 & 1 & 2 & 1.9 & 2.0 \\
\hline 4 & i & 0.188 & | & | & i & 1 & 2.2 & 1.2 \\
\hline $5^{*}$ & i & 0.188 & | & I & i & 2 & 2.8 & 0.65 \\
\hline 6 & i & 0.163 & 7.1 & I & i & 1 & 2.0 & 1.1 \\
\hline $7^{*}$ & i & 0.393 & 12.6 & 1 & i & 1 & 2.1 & 0.47 \\
\hline 8 & | & 1 & 4.6 & 1 & i & 2 & 0.85 & 7.2 \\
\hline 9 & i & | & 4.6 & 2.9 & 0.68 & 3 & 2.1 & 1.5 \\
\hline 10 & i & | & 7.1 & 3.1 & 0.72 & 2 & 1.9 & 2.3 \\
\hline $11^{*}$ & i & i & 1 & 3.4 & 0.79 & 1 & 2.3 & 0.45 \\
\hline 12 & 7 & | & | & 3.4 & 0.79 & 2 & 2.0 & 1.2 \\
\hline 13 & 1 & i & i & 2.9 & 0.68 & 1 & 2.1 & 2.1 \\
\hline 14 & 2 & i & i & 1 & 1 & i & 2.3 & 1.2 \\
\hline 15 & 9 & | & i & i & i & 2 & 2.0 & 1.9 \\
\hline 16 & 9 & | & i & i & i & 3 & 1.8 & 4.6 \\
\hline $17^{*}$ & 5 & | & 7.1 & I & 1 & 2 & 2.3 & 0.71 \\
\hline 18 & 1 & | & 4.6 & i & $i$ & 3 & 2.3 & 1.9 \\
\hline 19 & 1 & 0.393 & 4.6 & 2.9 & 0.68 & 2 & 1.2 & 2.4 \\
\hline \multicolumn{9}{|c|}{ (b) Overview and analysis of experimental data from Ref. [29]. } \\
\hline 20 & 9 & 0.393 & 4.6 & 2.9 & 0.68 & 2 & 0.78 & 4.8 \\
\hline 21 & | & | & | & 2.9 & 0.78 & 3 & 2.2 & 3.8 \\
\hline 22 & i & i & i & 3.1 & 0.72 & 2 & 2.4 & 2.4 \\
\hline 23 & i & i & i & 3.1 & 0.72 & 3 & 2.0 & 4.4 \\
\hline 24 & i & | & 1 & 3.4 & 0.79 & 2 & 1.9 & 3.0 \\
\hline 25 & 9 & 0.393 & 4.6 & 0.68 & 0.79 & 3 & 1.9 & 5.1 \\
\hline
\end{tabular}

\section{Theoretical analysis}

\subsection{Single degree of freedom oscillator model}

As proposed by Howe [36] for low Mach number flows, the acoustic field is described in terms of solutions of d'Alembert's equation for the total enthalpy fluctuation $B^{\prime}$. As the frequency of the oscillation is low compared to the cut-on frequency for transversal pipe modes, the acoustic field can be described in terms of traveling plane acoustic waves. One can also describe the acoustic field in terms of a series of standing waves [1].

Here the crudest approximation is used for which only one dominating standing wave is considered corresponding to the resonance frequency $f_{n}$. Furthermore the discussion is restricted to the limit cycle, which is assumed to be dominated by a single harmonic oscillation. This implies that the acoustic field described in terms of total enthalpy fluctuations $B^{\prime}$ is

$$
B^{\prime}=-\left|b_{n}\right| \cos \left(k_{n} x\right) \cos \left(\omega t+\Phi_{n}\right),
$$

where $k_{n}=2 \pi f_{n} / c=\omega_{n} / c$ is the wavenumber, $\omega=2 \pi f$ is the angular frequency, $\left|b_{n}\right|$ is the amplitude of the total enthalpy fluctuations and $x$ is the distance from the nozzle inlet. The phase $\Phi_{n}$ is chosen such that vortex shedding occurs at $t=0$.

For a one-dimensional flow of time averaged velocity $U$ and velocity fluctuation $u^{\prime}$, the total enthalpy fluctuation are related to the pressure fluctuation $p^{\prime}$ by

$$
B^{\prime}=\frac{p^{\prime}}{\rho_{0}}+u^{\prime} U
$$

where $\rho_{0}$ is the density of the unperturbed mean flow. The acoustic power flux (energy per unit time and unit surface area), also called the acoustic intensity $I$ is given by the following relation [36].

$$
I=m^{\prime} B^{\prime}
$$

where $m^{\prime}$ is the fluctuation in mass flux, i.e. one has

$$
m^{\prime}=\rho^{\prime} U+\rho_{0} u^{\prime} .
$$

The acoustic velocity $u^{\prime}$ is obtained by using the linearized equation of Euler in the form

$$
\frac{\partial u^{\prime}}{\partial t}=-\frac{\partial B^{\prime}}{\partial x}
$$


Using Eq. (7), one finds:

$$
u^{\prime}=-\frac{\left|b_{n}\right|}{c}\left(\frac{\omega_{n}}{\omega}\right) \sin \left(k_{n} x\right) \sin (\omega t) \text {. }
$$

As proposed by Culick [1] this system is described as a damped single degree of freedom harmonic oscillator driven by a source term $\mathcal{F}_{\text {vortex }}$

$$
\mathcal{M}_{\text {eff }} \frac{\mathrm{d}^{2} b_{n}}{\mathrm{~d} t^{2}}+\gamma_{\text {eff }} \frac{\mathrm{d} b_{n}}{\mathrm{~d} t}+K_{\text {eff }} b_{n}=\mathcal{F}_{\text {vortex }}
$$

The effective mass $\mathcal{M}_{\text {eff }}$ is defined, such that

$$
\frac{1}{2} \mathcal{M}_{\mathrm{eff}}\left(\frac{\mathrm{d} b}{\mathrm{~d} t}\right)^{2}
$$

corresponds to the kinetic energy in the acoustic standing wave, viz.

$$
\mathcal{M}_{\text {eff }}=\frac{4 \pi r_{p}^{2}}{\left(\omega_{n}\left|b_{n}\right|\right)^{2}}\left\langle\int_{0}^{L_{\text {eff }}} \frac{1}{2} \rho_{0}\left(u^{\prime}\right)_{\omega=\omega_{n}}^{2} d x\right\rangle=\frac{\rho_{0} \pi r_{p}^{2} L_{\text {eff }}}{2\left(\omega_{n} c\right)^{2}}
$$

where the brackets $\langle\cdots\rangle$ indicate time averaging over a period of oscillation. By definition the spring constant is related to the resonance frequency $\omega_{n}=2 \pi f_{n}$ by:

$$
K_{\text {eff }}=\mathcal{M}_{\text {eff }} \omega_{n}^{2}
$$

The time averaged acoustic power $\left\langle\mathcal{P}_{\text {diss }}\right\rangle$ dissipated at the limit cycle is given by:

$$
\left\langle\mathcal{P}_{\text {diss }}\right\rangle=\left\langle\gamma_{\text {eff }}\left(\frac{\mathrm{d} b}{\mathrm{~d} t}\right)^{2}\right\rangle
$$

where $\gamma_{\text {eff }}$ is the effective damping.

The acoustic energy produced by the vortex flow during the interaction with the nozzle is estimated from the energy corollary of Howe [37]:

$$
\left\langle\mathcal{P}_{\text {vortex }}\right\rangle=-\rho\left\langle\int_{V}(\boldsymbol{\omega} \times \mathbf{v}) \cdot \mathbf{u}^{\prime} d^{3} x\right\rangle
$$

by using a two-dimensional incompressible potential flow model assuming a line vortex $\omega=\left(0,0, \Gamma \delta\left(x-x_{\Gamma}\right) \delta\left(y-y_{\Gamma}\right)\right)$ for the vortex path traveling at a velocity $\mathbf{v}$ where the vortex position is $\left(x_{\Gamma}, y_{\Gamma}, 0\right)$. In Eq. (18), $\mathbf{u}^{\prime}$ denotes the local acoustic velocity.

\subsection{Linear damping}

The linear damping $\gamma_{\text {eff }}$ in Eq. (13) is the sum of losses due to sound radiation at the nozzle $\gamma_{\text {nozzle }}$, vortex shedding at the orifice $\gamma_{\text {orifice }}$ and viscous dissipation in the porous wall $\gamma_{\text {porous }}$ :

$$
\gamma_{\text {eff }}=\gamma_{\text {nozzle }}+\gamma_{\text {orifice }}+\gamma_{\text {porous }} \text {. }
$$

In the axial flow injection case, the visco-thermal damping along the pipe wall appears to be negligible compared to the other contributions. Models for these losses are described in Ref. [26] and are summarized in A.

\subsection{Vortex path}

The flow model used to predict the vortex path and its interaction with the local acoustic field at the nozzle inlet is a two dimensional incompressible potential flow model. For convenience the flow in a quarter of the channel cross-section is considered. The nozzle is represented as an abrupt contraction in a two dimensional channel from a square section of width $S_{1}=\sqrt{\pi} r_{p} / 2$ to a rectangular channel of height $S_{2}$ and width $S_{1}$ where $S_{2}$ is chosen following the formula:

$$
S_{2}=S_{1} M\left(\frac{\gamma+1}{2\left(1+\frac{\gamma-1}{2} M^{2}\right)}\right)^{\frac{\gamma+1}{2(\gamma-1)}}
$$

such that for an inlet Mach number $M$ in a section of area $S_{1}^{2}$ the channel area $S_{1} S_{2}$ after the 2-Dimensional contraction corresponds to the area of the chocked nozzle throat, as calculated using quasi-one dimensional steady flow gas dynamics [38].

The vortex path is calculated using the following simplifying assumptions:

1. The vortex does not interfere with other vortices that are shed earlier or later. 


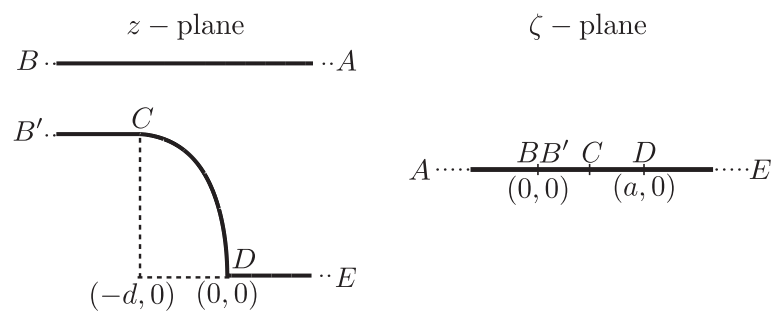

Fig. 4. Conformal mapping, Schwarz-Christoffel transformation of backward facing step with uniform approach flow. The inner points in $z$-plane are mapped to the upper two quadrants in the $\zeta$-plane.

2. A new vortex is shed each oscillation period when the acoustic velocity $u^{\prime}$ calculated with Eq. (12) vanishes at the orifice $x=x_{0}$ turning in the direction of the main flow towards the nozzle.

3. The vortex circulation $\Gamma$ increases linearly during the first oscillation period after it is shed, following the model of Eq. (5).

4. The vortex circulation $\Gamma$ remains constant after one period of oscillation.

5. The acoustic flow does not influence the vortex path.

6. The cavity surrounding the nozzle inlet does not influence the vortex path.

By using the conformal mapping in the complex plane $z=x+\mathrm{i} y=f(\zeta)$ where $\mathrm{i}^{2} \equiv-1$, the physical plane $(x, y)$ is mapped into the half plane above the real axis in the complex $\zeta$ plane as shown in Fig. 4.

A modified Schwarz-Christoffel transformation as proposed by Henrici is used [39]. Details of the transformation can be found in Ref. [40]. The Henrici transformation introduces the contraction length $d$, in the $z$ plane as shown in Fig. 4. Calculations are presented for a sharp square edge $d=0$ and for a rounded nozzle inlet with $d=S_{1} / 2$. This will provide some insight into the effect of the nozzle geometry on the sound production.

The solution for the potential of the flow is obtained with the method of images. The vortex velocity and path $z_{\Gamma}$ are then obtained by standard methods. Details are provided in Appendix B.

\subsection{Acoustic field}

The acoustic field in the pipe is approximated by a plane acoustic standing wave Eq. (12). A more detailed description of the local acoustic field in the vicinity of the nozzle inlet is needed when applying the energy corollary Eq. (18). By definition the acoustic field is a potential flow (frictionless). In the vicinity of the nozzle inlet there are two contributions to the acoustic velocity: the flow corresponding to the radiation of sound through the nozzle $\mathbf{u}_{n}^{\prime}$ and the flow $\mathbf{u}_{c}^{\prime}$ induced by the acoustic compression of the gas in the cavity surrounding the nozzle inlet. At low frequencies as considered here, one can assume a quasi-steady acoustic flow through the nozzle. This is in the incompressible potential flow model a harmonically oscillating flow induced by a line (2-D point) source positioned at $\zeta=0$ corresponding to points $B$ and $B^{\prime}$ in Fig. 4. The source strength $Q_{n}$ is given by:

$$
Q_{n}=\frac{\gamma-1}{2} M \frac{\left|b_{n}\right|}{c} S_{1} \cos \left(\omega t+\Phi_{n}\right),
$$

with scalar potential

$$
\phi_{n}=\frac{Q_{n}}{\pi} \ln (\zeta) .
$$

The acoustic field induced by the cavity is represented by a line source $Q_{c}$ of length $S_{1}$ positioned at the corner just before the contraction of the nozzle inlet, at point $D$ in Fig. 4. Fig. 4 shows the cross-sectional two dimensional plane out of which the line source protrudes. Point $D$ is at $z=0$ which corresponds to $\zeta=a$ (see $\mathrm{B}$ ). As the volume $V_{c}$ of the cavity is small compared to the acoustic wavelength one can assume a uniform pressure in the cavity and estimate $Q_{c}$ from an integral mass balance neglecting the steady flow velocity

$$
Q_{c}=-\frac{V_{c}}{4 S_{1} \rho_{0} c^{2}} \frac{\mathrm{d} p^{\prime}}{\mathrm{d} t}=-\frac{V_{c}}{4 S_{1} c^{2}} \frac{\mathrm{d} b_{n}}{\mathrm{~d} t}
$$

with scalar potential

$$
\phi_{c}=\frac{Q_{c}}{\pi} \ln (\zeta-a) .
$$


In order to investigate the effect of the geometry of the cavity opening, some calculations are carried out in which the volume flux $Q_{c}$ is distributed between two line sources, placed along the real axis in the $\zeta$-plane.

The acoustic velocity (in complex notation) in the $z$-plane is given by:

$$
u^{\prime}=\left[\frac{\mathrm{d}\left(\phi_{n}+\phi_{c}\right)}{\mathrm{d} z}\right]^{*}=\left[\frac{\mathrm{d}\left(\phi_{n}+\phi_{c}\right)}{\mathrm{d} \zeta} \frac{1}{\left(\frac{\mathrm{d} f}{\mathrm{~d} \zeta}\right)_{\zeta_{L}}}\right]^{*}
$$

where $(\ldots)^{*}$ denotes the complex conjugate.

\subsection{Energy balance}

The power $\mathcal{P}_{\text {vortex }}$ (Eq. (18)) generated by a line vortex of circulation $\Gamma$ of length $2 S_{1}$ and position $z_{\Gamma}$ is given in complex notation by Ref. [41].

$$
\mathcal{P}_{\text {vortex }}(t)=-4 S_{1} \operatorname{Re}\left[\mathrm{i} \rho_{0} \Gamma u_{\mathrm{ac}}^{*} \frac{\mathrm{d} z_{\Gamma}}{\mathrm{d} t}\right]=-4 S_{1} \operatorname{Re}\left[\mathrm{i} \rho_{0} \Gamma u_{\mathrm{ac}}^{*} \frac{\mathrm{d} \zeta_{\Gamma}}{\mathrm{d} t}\left(\frac{\mathrm{d} f}{\mathrm{~d} \zeta}\right)_{\zeta_{\Gamma}}\right] .
$$

As the vortex position $\zeta_{\Gamma}$ and its velocity $\mathrm{d} \zeta_{\Gamma} / \mathrm{d} t$ are known in the $\zeta$-plane as a function of the time the power generated by a vortex can be obtained. The time averaged power generated by the vortices is calculated as

$$
\left\langle\mathcal{P}_{\text {vortex }}\right\rangle=\frac{\omega}{2 \pi} \int_{0}^{t_{\max }} \mathcal{P}_{\text {vortex }}(t) \mathrm{d} t .
$$

where the origin of time is taken at the moment the vortex is shed from the orifice at $z_{0}$ and $t_{\max }$ is a time after which the vortex has passed the contraction and does not produce sound anymore, because its path is parallel to the acoustic streamlines in the channel. When there are more than one vortex between the orifice and the nozzle this integration time is larger than one period of oscillation $2 \pi / \omega$. The contribution of all vortices within one period of oscillation equals the contribution of a single vortex over its entire active life $0<t<t_{\max }$.

Assuming the oscillation frequency to correspond to the eigenfrequency of a mode $\omega=\omega_{n}$ the energy balance is given by

$$
\left\langle\mathcal{P}_{\text {vortex }}\right\rangle=\left\langle\gamma_{\text {eff }}\left(\frac{\mathrm{d} b_{n}}{\mathrm{~d} t}\right)^{2}\right\rangle
$$

where $\left\langle\mathcal{P}_{\text {vortex }}\right\rangle$ is calculated using Eq. (26) combined with Eq. (27). This yields a first order equation for the amplitude $\left|b_{n}\right|$ of the standing wave in the pipe. Indeed the energy losses scale with the square of the amplitude $\left|b_{n}\right|^{2}$ while the sound production scales with the amplitude $\left|b_{n}\right|$. This is a consequence of the assumption that, both the circulation $\Gamma$ and the vortex path are assumed to be independent of the amplitude of the acoustic perturbation. In the model, only the phase of the vortex shedding at the orifice is determined by the acoustic oscillation.

\subsection{Lumped model}

The energy balance does not allow the prediction of the variation of oscillation frequency with the Mach number $M$ as observed in Figs. 1 and 3. A lumped model is developed here for this purpose. Analogous to Culick [8] one can develop the solution $B^{\prime}$ of the aeroacoustic analogy in a series of orthogonal modes $\psi_{n}$ :

$$
B^{\prime}=\sum_{n} b_{n} \psi_{n}
$$

where $\psi_{n}$ is the solution of the Helmholtz equation

$$
\nabla^{2} \psi_{n}+k_{n} \psi_{n}=0
$$

with the boundary condition $\mathbf{n} \cdot \nabla \psi_{n}=0$ on a surface $S$ with outer unit normal $\mathbf{n}$ enclosing the combustion chamber. The wavenumber $k_{n}=\omega_{n} / c$ corresponds to the acoustic resonance frequency $\omega_{n}$. It can be shown that for such loss free boundary conditions, one has

$$
\int_{V} \psi_{n} \psi_{m} \mathrm{~d}^{3} x=E_{n}^{2} \delta_{n m},
$$

where $\delta_{n m}$ is the Kronecker delta and $E_{n}$ is a normalization factor. The volume $V$ is enclosed by the surface $S$.

For a low Mach number homentropic flow with main steady velocity field $\mathbf{U}_{\mathbf{0}}$ the analogy of Howe [36] can be written as

$$
\frac{1}{c^{2}} \frac{\partial^{2} B^{\prime}}{\partial t^{2}}-\nabla^{2} B^{\prime}=\nabla \cdot(\boldsymbol{\omega} \times \mathbf{v})-\frac{1}{\rho_{0}} \mathbf{U}_{0} \cdot \nabla\left(\frac{\partial B^{\prime}}{\partial t}\right) .
$$


The second term on the right hand side of Eq. (32) is usually neglected. It is, however, essential in order to take into account convective losses at the nozzle and porous wall. Using Green's function $G(\mathbf{x}, \mathbf{y}, t-\tau)$ developed in modes $\psi_{n}$ and neglecting second order terms in the Mach number, one finds a set of ordinary differential equations for the mode amplitudes:

$$
\frac{\mathrm{d}^{2} b_{n}}{\mathrm{~d} t^{2}}+\frac{\gamma_{\mathrm{eff}}}{\mathcal{M}} \frac{\mathrm{d} b_{n}}{\mathrm{eff}}+\omega_{n}^{2} b_{n}=-\left(\frac{c}{E_{n}}\right)^{2} \int_{V}(\boldsymbol{\omega} \times \mathbf{v}) \cdot \nabla_{\mathbf{y}} \psi_{n} \mathrm{~d}^{3} y
$$

In the derivation of Eq. (33) the volume integral

$$
\int_{-\Theta}^{\Theta} \int_{V} \nabla_{\mathbf{y}} G \cdot \mathbf{U}_{0} \frac{\partial \rho^{\prime}}{\partial \tau} \mathrm{d}^{3} y \mathrm{~d} \tau
$$

averaged over a long time interval $[-\Theta, \Theta]$ has been neglected. In the particular case considered here $\nabla_{\mathbf{y}} G$ scales with $\nabla \psi_{n}, \rho^{\prime}$ scales with $\psi_{n}$, and $\mathbf{U}_{0}$ is almost uniform along the pipe. Thus the volume integral is expected to vanish, because $\nabla_{\mathbf{y}} \psi_{n} \cdot \mathbf{U}_{\mathbf{0}} \sim$ $\sin \left(k_{n} y_{1}\right)$ while $\psi_{n} \sim \cos \left(k_{n} y_{1}\right)$.

For a stable limit cycle $b_{n}=\left|b_{n}\right| \cos \left(\omega t+\Phi_{n}\right)$, the phase $\Phi_{n}$ is chosen such that $t=0$ corresponds to the moment at which a new vortex is shed. Substitution in Eq. (33) and multiplying by $\sin \left(\omega t+\Phi_{n}\right)$ one obtains, by averaging over a period of oscillation:

$$
\left|b_{n}\right|=-\frac{2 \mathcal{M}_{\mathrm{eff}}}{\gamma_{\mathrm{eff}}}\left(\frac{c}{E_{n}}\right)^{2}\left\langle\int_{V}(\omega \times \mathbf{v}) \cdot \nabla_{\mathbf{y}} \psi_{n} \mathrm{~d}^{3} y \sin \left(\omega t+\Phi_{n}\right)\right\rangle .
$$

Substitution in Eq. (33) and multiplying by $\cos \left(\omega t+\Phi_{n}\right)$ and time averaging over one period of oscillation yields a second equation for $\left|b_{n}\right|$. Combining this second equation for $\left|b_{n}\right|$ with the one above, one finds

$$
\omega_{n}-\omega=\frac{\gamma_{\mathrm{eff}}}{\mathcal{M}_{\mathrm{eff}}\left(\omega+\omega_{n}\right)} \frac{\left\langle\int_{V}(\omega \times \mathbf{v}) \cdot \nabla_{\mathbf{y}} \psi_{n} \mathrm{~d}^{3} y \cos \left(\omega t+\Phi_{n}\right)\right\rangle}{\left\langle\int_{V}(\omega \times \mathbf{v}) \cdot \nabla_{\mathbf{y}} \psi_{n} \mathrm{~d}^{3} y \sin \left(\omega t+\Phi_{n}\right)\right\rangle} .
$$

When substituting the approximation $\omega=\omega_{n}$ in the first equation one obtains the same expression for $\left|b_{n}\right|$, as for the energy balance in the case where sound is only generated by the interaction of a vortex with the acoustic field generated by the cavity $V_{c}$ i.e. when sound production due to ingestion of a vortex by the nozzle is neglected.

The gradient of the eigenfunction $\nabla \psi_{n}$ accurately describes the acoustic flow in the combustion chamber including the effect of the cavity volume $V_{c}$. Due to the radiation free boundary condition $\mathbf{n} \cdot \nabla \psi_{n}=0$ this is a poor approximation for the local acoustic velocity field induced by radiation through the nozzle. However, as noted by Culick [8] the energy losses due to radiation are correctly described and included in $\gamma_{\text {eff }}$.

Substituting $\omega=\omega_{n}$ in the right hand side of Eq. (36), yields an estimate for the deviation $\omega_{n}-\omega$ of the oscillation frequency $\omega$ from the resonance frequency $\omega_{n}$. This approximation is used to obtain a theoretical value of the slope $(M / \omega) \mathrm{d} \omega / \mathrm{d} M$ of the frequency as a function of the Mach number $M$ around a pulsation maximum. A more accurate solution can be obtained by successive substitution of approximations of $\omega$ [8]. Only the initial approximation described above is considered here. It is obvious from this approximation that the slope is a measure for the quality factor $\mathcal{Q} \equiv \mathcal{M}_{\mathrm{eff}} \omega_{n} / \gamma_{\text {eff }}$ of the resonator.

\section{Results}

\subsection{Comparison of model with experiments}

Table 1 contains the nozzle numbers, as defined by Anthoine [17] and associated cavity volumes $V_{c}$, used in cold gas scale experiments carried out at the von Karman Institute (VKI).

In the following tables the experimental data are given for maxima of pulsation levels observed as a function of the Mach number. An overview of the pulsation maxima in all the available axial injection experiments is shown in Table 2 . For all the experiments the pipe radius $r_{p}$ is fixed at $r_{p}=3.8 \times 10^{-2} \mathrm{~m}$. In column 8 of Table 2 the estimated acoustic mode number $n$ of the experiments is shown. The estimated hydrodynamic mode number $m_{h}$ is calculated using Eq. (3). The results for $m_{h}$ are shown in column 9 of Table 2. It is noteworthy that most of the experiments are in the second hydrodynamic mode, for which $m_{h} \simeq 2$. This means that in most experiments two vortices, with a phase delay of a period of oscillation between them, are traveling simultaneously from the orifice to the nozzle. For measurements 8 and 19 (Table 2), which both have a small distance $L_{0}=4.6 \times 10^{-2} \mathrm{~m}$ between the orifice and the nozzle inlet, a hydrodynamic mode number $m_{h} \simeq 1$ is observed. This means that a single vortex is present between the orifice and the nozzle in these experiments.

The relative acoustic velocity fluctuation $u_{\text {rms }} / U$ is calculated with Eq. (4). The results are shown in column 10 of Table 2 . If $10^{-2} \leq u_{\mathrm{rms}} / U \leq 10^{-1}$, the measurement is in the moderate amplitude regime [30]. The model described in this paper is valid for this regime. If this relative fluctuation in acoustic velocity is $\mathcal{O}\left(10^{-3}\right)$, it is in the so-called low-amplitude regime [30], in which case the proposed model is not valid. These experiments are marked with an asterisk e.g. $5^{*}$. One will indeed observe that the theory fails for these experiments.

The influence of the cavity volume $V_{c}$ used in experiments is shown in Table 3. All experiments in Table 3 have a fixed pipe length $L_{p}=0.393 \mathrm{~m}$, distance between the orifice and the nozzle inlet $L_{o}=7.1 \times 10^{-2} \mathrm{~m}$, orifice radius $r_{o}=2.9 \times 10^{-2} \mathrm{~m}$ and vena contracta factor $\Upsilon=0.68$. 
Table 3

Influence of cavity volume $V_{c}$ for fixed pipe length $L_{p}=0.393 \mathrm{~m}$, orifice length $L_{o}=7.1 \times 10^{-2} \mathrm{~m}$, orifice radius $r_{o}=2.9 \times 10^{-2} \mathrm{~m}$ and vena contracta factor $\Upsilon=0.68$. In this table repeating values in columns are replaced by a dashed line.

\begin{tabular}{llllllll}
\hline Num. & Nozzle & $V_{c}\left(\mathrm{~m}^{3}\right) \times 10^{-5}$ & $n$ & $M_{\exp } \times 10^{-2}$ & $\alpha\left(\frac{p_{\text {rms }}}{p}\right)_{\exp } \times 10^{-3}$ & $\frac{M_{\text {th }}}{M_{\exp }}$ & $\frac{\left(p_{\text {rms }}\right)_{\text {th }}}{\left(p_{\text {rms }}\right)_{\exp }}$ \\
\hline 1 & 7 & 18.4 & 2 & 8.0 & 2.0 & 0.94 & 1.0 \\
13 & 1 & 20.3 & $\mid$ & 7.9 & 2.2 & 0.95 & 0.91 \\
14 & 2 & 10.0 & $\mid$ & 7.8 & 2.4 & 1.0 & 0.93 \\
15 & 9 & 21.8 & $\mid$ & 8.6 & 1.9 & 0.87 & 1.0 \\
$17^{*}$ & 5 & 6.71 & 2 & 7.9 & 2.2 & 1.0 & 1.0 \\
2 & 7 & 18.4 & 3 & 14 & 9.3 & 0.83 & 1.1 \\
16 & 9 & 21.8 & 3 & 14 & 17 & 0.81 & 0.69 \\
\hline
\end{tabular}

It is expected that the relative pressure pulsations are proportional to the cavity volume $V_{c}$ used in the experiment [17]. Thus the experimental data are scaled by the cavity volume used for the experiment. The pulsation amplitude $\left(p_{\mathrm{rms}} / p\right)_{\exp }$ is multiplied by the factor:

$$
\alpha \equiv \frac{V_{\text {nozzle } 7}}{V_{c}} .
$$

In column 3 of Table 1 the scaling factor $\alpha$ is shown for all nozzles considered in this text. The resulting scaled relative pressure pulsations are shown in column 6 of Table 3.

In the last two columns of Table 3 the experiments are compared to model, using the fit parameter $K=1 / 3$. To determine the pulsation maximum predicted using the energy balance model the following steps are taken. An initial estimate of the Mach number is taken at an arbitrary number near the Mach number that produced a pressure oscillation maximum $\left(p_{\text {rms }} / p\right)_{\exp }$ in the experiments. The relative pulsation amplitude $\left(p_{\mathrm{rms}} / p\right)_{\text {th }}$ is calculated using the energy balance. The Mach number is increased by an increment, then the relative pulsation amplitude is again calculated using the energy balance. This is repeated until a maximum in relative pulsation amplitude is reached. If none is reached by systematic increase the same process is done with a decreasing increment in Mach number starting from the initial estimated Mach number.

In Table 3 one observes that, experiments taken from Ref. [17] are reproduced with the model for $n=2$ within $10 \%$, for both Mach numbers and relative pressure pulsation amplitudes. For $\mathrm{n}=3$ the deviation of $30 \%$ between the model and experiments is comparable to the deviation between experiments using different nozzles.

Fig. 5 shows the measurements from Anthoine [17] as a function of the Mach number for the second acoustic mode $n=2$. The upright triangles indicate data obtained with nozzle 1, the circles nozzle 2, squares correspond to data for nozzle 7 and sideways triangles nozzle 9 . These data are scaled using the multiplication factor $\alpha=V_{\text {nozzle }} 7 / V_{c}$ since a proportionality of the pressure pulsations to $V_{c}$ is expected [17]. This scaling allows to collapse the data to some extent. One observes a deviation of approximately $20 \%$ between the maxima of the pulsation amplitude.

To investigate the influence of the cavity volume $V_{c}$ used by the model two lines are shown in Fig. 5. The solid line corresponds to the model with nozzle 7 for which the scaling factor is $\alpha=1$. The dashed line corresponds to the model with nozzle 2 , $\alpha=V_{\text {nozzle7 }} / V_{\text {nozzle2 }}$. Both model predictions are made with $K=1 / 3$. Both predict the pressure pulsation quite well. The Mach

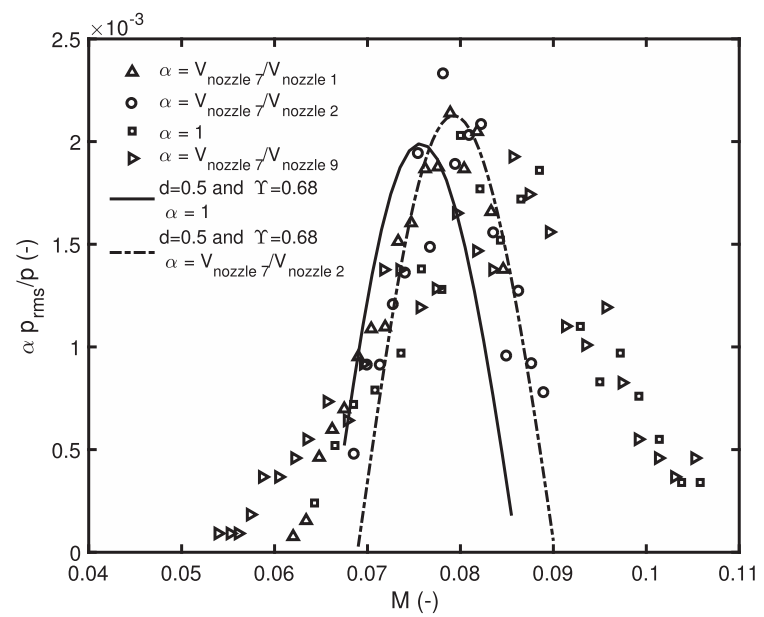

Fig. 5. Influence of cavity volume $V_{c}$ on the rms pressure as a function of the Mach number for $n=2$. 
number of the maximum is reproduced within $10 \%$ and the maximum pressure pulsation amplitude is reproduced within $10 \%$.

In Fig. 5, one observes a shift in Mach number where the maximum relative pressure pulsation (where $f=f_{n}$ ) occurs for the model. This is due to the fact that at the pulsation maximum the hydrodynamic mode number $m_{h}$ is fixed. As $V_{\text {nozzle }}>V_{\text {nozzle2 }}$ the effective length $L_{\text {eff }}$ for nozzle 7 is larger than for nozzle 2 . Thus the resonance frequency $f_{n}$, which is inversely proportional to $L_{\text {eff }}$, for nozzle 7 is lower than for nozzle 2 . Hence, the Mach number predicted for the maximum pressure pulsation is lower for nozzle 7 than for nozzle 2, which keeps $m_{h}=f_{n} L_{0} /(0.4 c M)$ constant. Note that the predicted shift is not observed in the experiments.

Fig. 6 shows scaled data obtained by Anthoine [17] for the third estimated acoustic mode $n=3$. In this figure squares indicate data obtained with nozzle 7 and sideways triangles those found for nozzle 9. The solid line in Fig. 6 are predictions obtained using the model, with $V_{c}=V_{\text {nozzle } 7}$ and $K=1 / 3$. Both for the empirical data and the model prediction, the dimensions are $L_{p}=0.393 \mathrm{~m}, L_{0}=0.071 \mathrm{~m}, r_{o}=0.029 \mathrm{~m}$ and $\Upsilon=0.68$.

Looking at Fig. 6, one observes that the empirical data for $n=3$ do not collapse, there is at least a $50 \%$ deviation between the points obtained with nozzle 7 and nozzle 9 respectively. The model predicts an intermediate amplitude and a rather low Mach number $M_{\text {th }} \simeq 0.116$ compared to the Mach of the measurements $M_{\exp }$ for the largest amplitudes.

The influence of the pipe length $L_{p}$ used in the experiments is investigated in Table 4 . The results in Table 4 were obtained using nozzle 7 for a fixed distance between the orifice and the nozzle inlet $L_{o}=7.1 \times 10^{-2} \mathrm{~m}$ and orifice radius $r_{o}=2.9 \times 10^{-2} \mathrm{~m}$ for which $\Upsilon=0.68$. The Mach numbers observed in the experiments are predicted by the model developed in this work within $20 \%$. For moderate amplitudes the relative pressure pulsations $p_{\text {rms }} / p$ are reproduced within a factor 2 when the factor $K=1 / 3$ is chosen to match the first experiment. When $K$ is varied around this fit value one observes the predicted amplitude to be linearly proportional to $K$. Note furthermore that for values of $K$ close or equal to $K=1$ the model can display unphysical behaviour, the vortex is not ingested by the nozzle when the circulation becomes too large. This effet is not only observed for a point vortex. It was also observed for a more realistic vorticity distribution by Hulshoff et al. [42]. Experimental results indicated with an asterisk fall in the so-called low amplitude regime [30]. The proposed model is not valid in this regime and the model drastically overestimates the pulsation levels. As indicated earlier similar behaviour is reported by Gallier et al. [21] in detailed numerical flow simulations of hot gas experiments. The slope $((M / f) \mathrm{d} f / \mathrm{d} M)_{\exp }$ determined from the experiments is reproduced within $40 \%$ by the model. The fair prediction of the slope indicates that the losses are well modelled by Eq. (36).

In Table 5, results are shown for cases where the distance between the orifice and the nozzle inlet $L_{0}$ is varied and for experiments carried out using nozzles 1,7 and 9 . The pipe length, the orifice radius were both respectively fixed at $L_{p}=0.393 \mathrm{~m}$ and $r_{o}=2.9 \times 10^{-2} \mathrm{~m}$ for which $\Upsilon=0.68$. All Mach numbers are reproduced within $25 \%$ using the model described in this study.

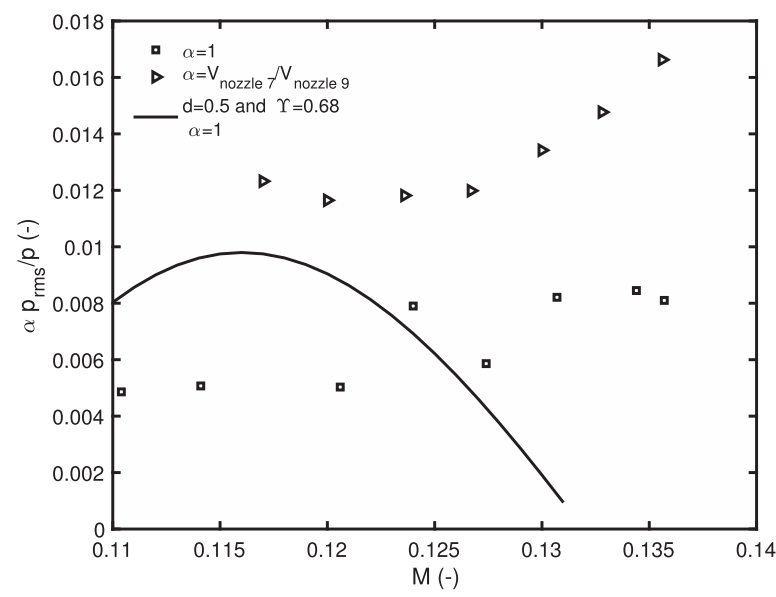

Fig. 6. Influence of nozzle on the rms pressure as a function of the Mach number for $n=3$.

Table 4

Influence of pipe length $L_{p}$.

\begin{tabular}{lllllllll}
\hline Num. & $n$ & $L_{p}(m)$ & $M_{\exp } \times 10^{-2}$ & $\left(\frac{p_{\text {rms }}}{p}\right)_{\exp } \times 10^{-3}$ & $\frac{M_{\text {th }}}{M_{\exp }}$ & $\frac{\left(p_{\text {rms }}\right)_{\text {th }}}{\left(p_{\text {rms }}\right.}$ & $\left(\frac{M}{f} \frac{\mathrm{d} f}{\mathrm{~d} M}\right)_{\exp }$ & $\left(\frac{M}{f} \frac{\mathrm{d} f}{\mathrm{~d} M}\right)_{\text {th }}$ \\
\hline 1 & 2 & 0.393 & 8.0 & 2.0 & 0.94 & 1.0 & 0.20 & 0.16 \\
2 & 3 & 0.393 & 14 & 9.3 & 0.83 & 1.1 & 0.12 & 0.07 \\
3 & 2 & 0.305 & 11 & 3.7 & 0.88 & 1.1 & 0.17 & 0.14 \\
4 & 1 & 0.188 & 7.8 & 1.3 & 0.97 & 1.3 & 0.31 & 0.28 \\
$5^{*}$ & 2 & 0.188 & 12 & 4.4 & 1.0 & 4.0 & 0.25 & 0.16 \\
6 & 1 & 0.163 & 9.8 & 1.6 & 0.88 & 1.8 & 0.39 & 0.30 \\
\hline
\end{tabular}


Table 5

Influence of the distance between the orifice and nozzle inlet $L_{0}$. In this table repeating values in columns are replaced by a dashed line.

\begin{tabular}{|c|c|c|c|c|c|c|c|c|}
\hline Num. & nozzle & $V_{c}\left(\mathrm{~m}^{3}\right) \times 10^{-5}$ & $n$ & $L_{o}(\mathrm{~m}) \times 10^{-2}$ & $M_{\text {exp }} \times 10^{-2}$ & $\alpha\left(\frac{p_{\mathrm{rms}}}{p}\right)_{\exp } \times 10^{-3}$ & $\frac{M_{t h}}{M_{\exp }}$ & $\frac{\left(p_{\text {rms }}\right)_{t h}}{\left(p_{\text {rms }}\right)_{\exp }}$ \\
\hline 18 & 1 & 20.3 & 3 & 4.6 & 7.3 & 2.0 & 0.93 & 0.81 \\
\hline 19 & 1 & 20.3 & 2 & 4.6 & 9.0 & 2.8 & 1.1 & 1.1 \\
\hline 1 & | & | & 2 & 7.1 & 8.0 & 2.0 & 0.94 & 1.0 \\
\hline 2 & i & i & 3 & 7.1 & 14 & 9.3 & 0.83 & 1.1 \\
\hline 15 & 9 & 21.8 & 2 & 7.1 & 8.5 & 1.9 & 0.87 & 1.0 \\
\hline 16 & 9 & 21.8 & 3 & 7.1 & 14 & 17 & 0.81 & 0.69 \\
\hline
\end{tabular}

In column 1 the number of the experiment is shown. For points in the moderate amplitude regime [30] the relative pressure pulsations $p_{\text {rms }} / p$ are reproduced within $60 \%$. The low amplitude experiment $7^{*}$ is over estimated by a factor 3 , when using the moderate amplitude model.

The influence of the orifice radius $r_{0}$ has been investigated. Although the orifice radius $r_{o}$ has some influence on the predictions, all predictions agree with Anthoine [17] and Yildiz [29] experiments within a similar measure of accuracy as the data described above.

\subsection{Predictions of the model}

Now that it has been shown that the model predicts moderate amplitude pulsations in axial injection experiments fairly well, it is used to investigate the influence of modifications in nozzle geometry. Results are presented in Fig. 7. The dotted line is for a contraction with a sharp square edge $(d=0)$ with the source concentrated in a single point at $z=0$. The solid line is for a smooth contraction $\left(d / S_{1}=0.5\right)$ with the source concentrated in a single point at $z=0$. The dashed line is for a smooth contraction for which the source is spread out viz. half the cavity volume is placed at $z=0$ and the other half on a point of the contraction for which $\operatorname{Im}\{z\}=0.25 S_{1}$. Spreading out the cavity volume between these two points simulates the finite size of the opening of the nozzle cavity. The sharpness of the contraction displaces the Mach number of the predicted maximum pressure pulsation to the left. It deviates approximately $5 \%$ from the maximum Mach number for the smoothed cases. There is however no appreciable impact on the predicted pressure pulsation amplitude for all the geometric variations. This leads one to conclude that the exact geometry of the nozzle does not have a major influence on the generation of relative pressure pulsation in SRMs.

Another interesting result of the model is that it allows for comparison between the sound produced without cavity $V_{c}=0$ and sound produced with a nozzle cavity present. This is illustrated in Fig. 8. One observes that the interaction with the cavity is two orders of magnitude more than the ingestion by the nozzle. Indeed, experiments carried out by Anthoine [17] with $V_{c}=0$ did not show significant pulsation amplitudes.

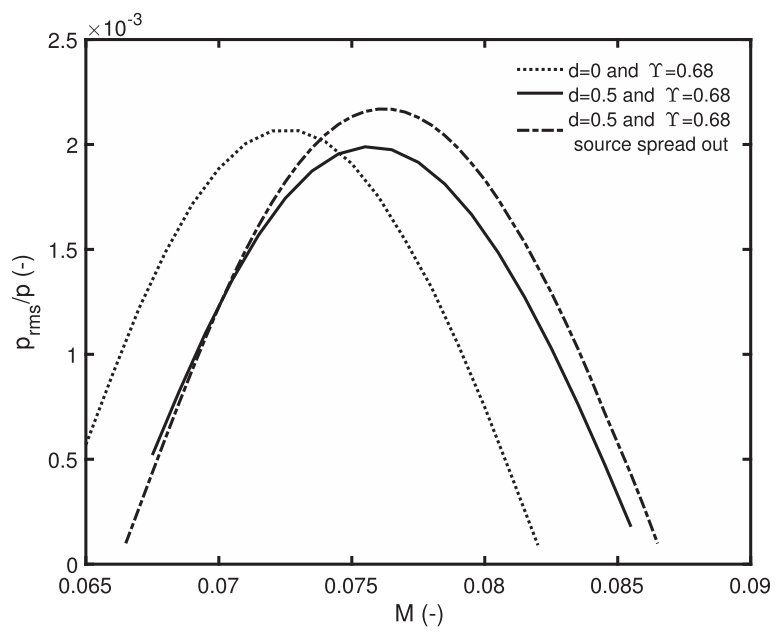

Fig. 7. Influence of geometric parameters of the nozzle on model predictions. 


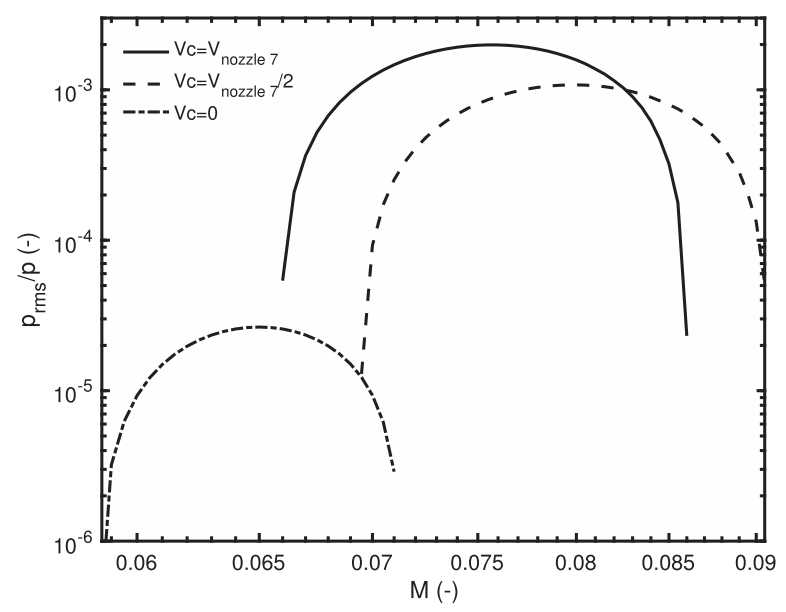

Fig. 8. Influence of the cavity volume $V_{c}$.

\section{Conclusion}

An analytical model based on an energy balance has been proposed to predict amplitudes of pulsations in a cold gas model of a SRM with vortex shedding at a thermal inhibitor and axial flow injection. The acoustic energy losses are described by linear quasi-steady models for acoustic radiation at the nozzle, vortex shedding at the inhibitor and viscous dissipation in the porous wall used for injection. This model predicts losses increasing quadratically with the pulsation amplitude. These losses are balanced by the sound production due to the interaction of vortices shed at the inhibitor with the critical nozzle at the downstream end of the SRM.

The magnitude of the circulation $\Gamma$ of the vortices is estimated as a fraction $K$ of the vorticity shed within one period of oscillation concentrated in a line vortex. The acoustic mode number $n$ is a user provided input to the model. The oscillation frequency $f$ is assumed to be equal to the corresponding eigenfrequency $f_{n}$.

The factor $K=1 / 3$ is fitted to one of the experimental results and it is supposed to account for the effect of turbulence in the flow and other differences between the model and the actual flow. Previous numerical simulations indicate that including turbulence reduces the predicted pulsations by about a factor 2, compared to a laminar flow simulation.

The dynamics of the vortex is described by a planar incompressible potential flow model. It is assumed that the acoustic field only determines the moment (phase) of vortex shedding at the inhibitor. The vortex circulation and path are independent of the amplitude of pulsation. The acoustic power generated by the vortex is calculated using Howe's energy corollary. This power scales linearly with the pulsation amplitude. This allows for a prediction of the pulsation amplitude for moderate amplitudes $10^{-2} \leq\left|u^{\prime}\right| / U \leq 10^{-1}$.

At lower amplitudes $\left|u^{\prime}\right| / U<10^{-2}$ the model severely overestimates the pulsation level. Predicting low amplitude pulsations appears to be difficult even when using sophisticated numerical models [21]. At low amplitude the vortex circulation becomes amplitude dependent and if the linear regime is approached the power predicted by Howe's corollary scales quadratically with the amplitude. Hence, the balance between acoustic losses and vortex sound generation does not allow to predict an amplitude. A minor increase in losses can reduce pulsation levels by orders of magnitude.

In the moderate amplitude regime the model predicts data of Anthoine et al. [17,29] quite well. The pulsation amplitudes are predicted within a factor two and the Mach number for the maximum pulsation level within $20 \%$ for orifice position $L_{0} / r_{p}=1.9$. For $L_{o} / r_{p}=1.2$ the model predicts the pulsation levels within a factor 3 and the Mach number within a factor 2 .

Applying the theory of Culick [8] to the Vortex Sound Analogy of Howe [36,43] one can obtain the Mach number dependence of the oscillation frequency around a pulsation maximum. The slope $(M / f) \mathrm{d} f / \mathrm{d} M$ is determined by the losses. Comparison between theory and experiments indicates that larger deviations are due to inaccuracies in the source model, in particular the description of the vortex flow, rather than inaccuracies in the losses.

An important result [17] is that the pulsation amplitude is proportional to the volume $V_{c}$ of the cavity around the inlet of the integrated nozzle. The model confirms this and predicts that pulsation amplitudes generated by the ingestion of the vortex are two order of magnitude lower than those generated by the interaction of the nozzle with the cavity.

The model, also, indicates that there are only minor differences as a result of the use of a nozzle with a square sharp edge nozzle inlet compared to a rounded nozzle inlet. A variation in position of the nozzle cavity inlet has only a small effect on the pulsation amplitude. This illustrates that a model such as this one provides insight in the influence of geometric parameters on the pulsation levels.

A correction to the model taking into account the axis-symmetrical geometry of the vortices and the effect of turbulence should be considered as a next step. This would involve sophisticated numerical simulations. Although the present model is crude it is almost completely analytical and well suited for parametric analysis. 


\section{Acknowledgements}

This work was funded through a CIFRE grant [Nr. 2015/0938] by ANRT and ArianeGroup for which the authors are grateful. Special thanks to Mr. Serge Radulovic of ArianeGroup for his support.

\section{Appendix A. Loss models} [26].

This appendix contains a summary of the loss models used for the model. More detail on these models can be found in Ref.

\section{Appendix A.1. Nozzle radiation}

The losses due to the radiation of the plane waves at the nozzle is estimated by using the quasi-steady isentropic theory of Marbel and Candel [24]. The key idea is that in this approximation the Mach number at the nozzle inlet is fixed by the geometry as long as the nozzle remains chocked. This implies for the fluctuations $c^{\prime}$ in speed of sound $c$ at the nozzle inlet:

$$
\frac{c^{\prime}}{c}=\frac{u^{\prime}}{U} \text {. }
$$

Using the isentropic gas relations for a perfect gas, combined with $p^{\prime}=c^{2} \rho^{\prime}=\left(p^{+}+p^{-}\right)$and $u^{\prime}=\left(p^{+}-p^{-}\right) /\left(\rho_{0} c\right)$, one finds the pressure reflection coefficient:

$$
R_{\text {nozzle }}=\frac{p^{-}}{p^{+}}=\frac{1-\frac{\gamma-1}{2} M}{1+\frac{\gamma-1}{2} M} .
$$

This is the result of Marble and Candel [24]. Thus the stagnation enthalpy reflection coefficient is

$$
\frac{B^{-}}{B^{+}}=R_{\text {nozzle }} \frac{1-M}{1+M}
$$

and the time averaged power losses $\left\langle\mathcal{P}_{\text {nozzle }}\right\rangle$ due to this radiation are given by

$$
\left\langle\mathcal{P}_{\text {nozzle }}\right\rangle=\frac{\rho_{0}\left|b_{n}\right|^{2}}{8 c}\left[1-R_{\text {nozzle }}^{2}\left(\frac{1-M}{1+M}\right)^{2}\right] \pi r_{p}^{2},
$$

where it is assumed that $\left|B^{+}\right|=\left|B^{-}\right|=\left|b_{n}\right| / 2$ in accordance with the assumption that the acoustic field is dominated by a standing wave, described by Eq. (7). Alternatively, based on Eq. (13), the nozzle radiation can be written in terms of $\gamma_{\text {nozzle }}$ as

$$
\left\langle\mathcal{P}_{\text {nozzle }}\right\rangle=\frac{\gamma_{\text {nozzle }}}{2} \omega^{2}\left|b_{n}\right|^{2},
$$

from which the nozzle damping coefficient is deduced

$$
\gamma_{\text {nozzle }}=\frac{\rho_{0} \pi r_{p}^{2}}{4 \omega^{2} c}\left[1-R_{\text {nozzle }}^{2}\left(\frac{1-M}{1+M}\right)^{2}\right] .
$$

\section{Appendix A.2. Vortex shedding at the orifice}

Due to flow separation at the sharp edges of the orifice a free jet will be formed downstream of the orifice with a smaller radius $r_{\text {jet }}$ than the orifice radius $r_{o}$. The ratio $\left(r_{\text {jet }} / r_{o}\right)^{2} \equiv \Upsilon$ is called the vena contracta factor. It is a function of $r_{o} / r_{p}$. Empirical data by Gilbarg [27] is used to determine this vena contracta factor in Section 2. Neglecting the pressure recovery downstream of the orifice one has a pressure in the free jet equal to the downstream acoustic pressure. This implies a loss of total acoustical enthalpy $B^{\prime}$ across the orifice given, for a quasi-steady flow, by

$$
\Delta B_{\text {orifice }}^{\prime}=u_{\text {jet }}^{\prime} U_{\text {jet }}-u^{\prime} U=u^{\prime} U\left[\left(\frac{r_{p}}{r_{\text {jet }}}\right)^{4}-1\right]
$$

where $u^{\prime} U$ is evaluated just upstream of the orifice. Terms of order $M^{2}$ are neglected and it is assumed that the surface averaged velocities $u^{\prime}$ and $U$ are equal upstream and downstream of the orifice.

The corresponding acoustic power losses are given by

$$
\left\langle\mathcal{P}_{\text {orifice }}\right\rangle=\left\langle m^{\prime} \Delta B_{\text {orifice }}^{\prime}\right\rangle \pi r_{p}^{2} \text {. }
$$

From which, in a similar way as for the nozzle radiation, one finds:

$$
\gamma_{\text {orifice }}=\frac{\rho_{0} \pi r_{p}^{2}}{\omega^{2} c}\left(\frac{\omega_{n}}{\omega}\right) M\left[\left(\frac{r_{p}}{r_{\text {jet }}}\right)^{4}-1\right] \sin ^{2}\left(k_{n} x_{0}\right),
$$


where the orifice position is $x_{o}=L_{o}+V_{c} / \pi r_{p}^{2}$.

Appendix A.3. Viscous damping at the porous wall

A quasi-steady isothermal flow through the porous wall is assumed. By continuity the mass flux $m=\rho_{0} u$ remains independent of the position $x$ in the porous wall. As for $M^{2}<<1$ the temperature remains uniform in the wall and the viscosity $\mu$ of the air is a function of the temperature only (ideal gas), one finds that the Reynolds number $R e_{d}=\rho_{0} u \bar{d} / \mu$ based on the characteristic pore width $\bar{d}$ is constant in the porous wall. The pressure gradient in the wall is determined by a friction coefficient $C_{D}$, which is assumed to be a function of $R e_{d}$ only [23]:

$$
\frac{\mathrm{d} p}{\mathrm{~d} x}=-\frac{\left(\rho_{0} u\right)^{2}}{\rho_{0} \bar{d}} C_{D}\left(R e_{d}\right)
$$

As $\rho_{0}=p /(R T)$ with $T$ the temperature and $R$ the specific gas constant one finds by integration $p_{r}^{2}-p^{2}=C\left(\rho_{0} u\right)^{2}$, with $C$ a constant. Neglecting the acoustic perturbations in the high pressure reservoir and using $\rho^{\prime} / \rho_{0}=p^{\prime} / p$ for isothermal flow, one finds:

$$
\frac{p^{\prime}}{p}=\left[1-\left(\frac{p_{r}}{p}\right)^{2}\right]\left(\frac{p^{\prime}}{p}+\frac{u^{\prime}}{U}\right)
$$

Using $p^{\prime}=p^{+}+p^{-}, \rho^{\prime}=p^{\prime} / c^{2}, u^{\prime}=\left(p^{+}-p^{-}\right) /\left(\rho_{0} c\right)$, after some algebra, one finds

$$
\gamma_{\text {porous }}=\frac{\rho_{0} \pi r_{p}^{2}}{4 \omega^{2} c}\left[1-R_{\text {porous }}^{2} \frac{(1+M)^{2}}{(1-M)^{2}}\right]
$$

with

$$
R_{\text {porous }}=\frac{p^{+}}{p^{-}}=\frac{\left(p_{r} / p\right)^{2}(1-\gamma M)-1}{\left(p_{r} / p\right)^{2}(1+\gamma M)-1} .
$$

\section{Appendix B. Vortex path}

The conformal mapping of Henrici [39] used to calculate the vortex path is

$$
z=\frac{S_{1}}{\pi}\left\{\alpha\left[\ln \left(\frac{1+\theta}{1-\theta}\right)-\ln \left(\frac{b+\theta}{b+\theta}\right)\right]+\beta \ln \left(\frac{\zeta}{b^{2}}\right)\right\}
$$

with

$$
\theta^{2}=\frac{\zeta-b^{2}}{\zeta-1}
$$

and

$$
\begin{aligned}
& \alpha=\frac{S_{1}-S_{2}}{\pi} \frac{b}{(b-1)} \\
& \beta=\frac{b S_{2}-S_{1}}{\pi(b-1)} .
\end{aligned}
$$

The parameter $b\left(b^{2} \equiv a\right)$ is determined by solving the implicit equation:

$$
2 \frac{\left(b S_{2}-S_{1}\right)}{\pi(b-1)} \ln b=d
$$

where $d$ is the length of the contraction (in the $z$ plane). These developments are used to provide some insight into the effect of the nozzle geometry on the sound production.

The main flow is described by a volume (line) sink placed at the origin $\zeta=0$ with strength $Q_{1}=-S_{1} U_{1}$ where $U_{1}=M c$ with $c$ the speed of sound in the pipe. The potential $\Phi_{0}$ describing this main flow is $\Phi_{0}=\left(Q_{1} / \pi\right) \ln (\zeta)$. The vortex path $\zeta_{\Gamma}$ in the $\zeta$ plane is obtained by integration in time of the equation,

$$
\frac{\mathrm{d} \zeta_{\Gamma}}{\mathrm{d} t}=\frac{1}{|\mathrm{~d} f / \mathrm{d} \zeta|^{2}}\left[-\frac{U_{1} S_{1}}{\pi \zeta_{\Gamma}}+\mathrm{i} \frac{\Gamma}{2 \pi\left(\zeta_{\Gamma}-\zeta_{\Gamma}^{*}\right)}+\mathrm{i} \frac{\Gamma}{4 \pi} \frac{\left(\mathrm{d}^{2} f / \mathrm{d} \zeta^{2}\right)_{\zeta_{\Gamma}}}{(\mathrm{d} f / \mathrm{d} \zeta)_{\zeta_{\Gamma}}}\right]^{*}
$$

where * indicates the complex conjugated value. The first term is the convection of the vortex by the main flow. The second term is the velocity induced by the image of the vortex. The mirror is the real axis of the $\zeta$ plane. The third term is the so-called 
Routh's correction for the self-induced velocity of the vortex [41,44]. The time integration is performed by means of the fourth order Runge-Kutta integration as proposed by Howe [43].

The initial position of the vortex is $z_{0}=x_{0}+\mathrm{i} h_{0}$ where $x_{0}=L_{0}$ is the position of the orifice and the height $h_{0}$ is chosen such that the area $4 S_{1}\left(S_{1}-h_{0}\right)$ of the "ring" in the 2-D representation is equal to the actual vortex ring surface $\pi r_{\text {jet }}^{2}$. The corresponding value $\zeta_{L}(0)$ is calculated by solving Eq. (B.1).

Once $\zeta_{L}(t)$ has been determined by numerical integration the corresponding vortex path in the physical plane $z_{\Gamma}$ is calculated using the explicit transformation Eq. (B.1).

\section{References}

[1] F.E.C. Culick, Combustion instabilities in solid propellant rocket motors, in: Internal Aerodynamics in Solid Rocket Propulsion RTO-EN-023, RTO/NATO, 2004.

[2] J. Anthoine, Solid Propellant Pressure Oscillations, STO-EN-AVT-206, RTO/NATO, 2014.

[3] Y. Fabignon, J. Anthoine, D. Davidenko, R. Devillers, J. Dupays, D. Gueyffier, Hijlkema, N. Lupoglazoff, J.M. Lamnet, L. Tessé, A. Guy, C. Erades, Recent advances in research on solid rocket propulsion, Journal AerospaceLab 11 (June 2016) 1-14.

[4] D.R. Greatrix, Scale effects on solid rocket combustion instability behaviour, Energies 4 (1)(2011) $90-107$.

[5] G.A. Flandro, S.R. Fischbach, J. Majdalani, Nonlinear rocket motor stability prediction: limit amplitude, triggering, and mean pressure shift, Phys. Fluids 19 (9) (2007) 094101-094116.

[6] F. Vuillot, Vortex- shedding phenomena in solid rocket motors, J. Propul. Power 11 (4) (1995) 626-639.

[7] F. Vuillot, G. Casalis, Motor flow instabilities - part 1, in: Internal Aerodynamics in Solid Rocket Propulsion RTO-EN-023, NATO-RTO, 2004.

[8] F.E.C. Culick, Unsteady Motions in Combustion Chambers for Propulsion Systems, volume RTO-AG-AVT-039 of RTO AGARDograph, RTO/NATO, 2006.

[9] G.A. Flandro, J. Majdalani, Aeroacoustic instability in rockets, AIAA J. 41 (3) (2003) 485-497.

[10] S.R. Fischbach, J. Majdalani, Volume-to-surface reduction of vorticoacoustic stability integrals, J. Sound Vib. 321 (3-5) (2009) 1007-1025.

[11] R.S. Brown, R. Dunlap, S. Young, R.C. Waugh, Vortex shedding as a source of acoustic energy in segmented solid rockets, J. Spacecraft 18 (4) (1981) 312-319.

[12] K.W. Dotson, S. Koshigoe, K.K. Pace, Vortex shedding in a large solid rocket motor without inhibitors at the segmented interfaces, J. Propul. Power 13 (2) (1997) 197-206.

[13] G. Casalis, F. Vuillot, Motor flow instabilities - part 2: intrinsic linear stability of the flow induced wall injection, in: Internal Aerodynamics in Solid Rocket Propulsion RTO-EN-023, NATO-RTO, 2004.

[14] D. Laboureur, B. Tóth, J. Anthoine, Investigation of the Taylor-Culick flow through particle image velocimetry and numerical simulation, AIAA J. 48 (6) (2010) 1077-1084.

[15] Y. Fabignon, J. Dupays, G. Avalon, F. Vuillot, N. Lupoglazoff, G. Casalis, M. Prévost, Instabilities and pressure oscillations in solid rocket motors, Aero. Sci. Technol. 7 (3) (2003) 191-200.

[16] P. Kuentzmann, V. Yang, J.W. Murdock, W.A. Johnston, G. Lengelle, J. Duterque, J.F. Trubert, M.W. Beckstead, M. Salita, F. Vuillot, J.F. Guery, F.E.C. Culick, Internal Aerodynamics in Solid Rocket Propulsion, RTO EDUCATIONAL NOTES EN-023 AVT-096, RTO NATO, 2004.

[17] J. Anthoine, Experimental and Numerical Study of Aeroacoustic Phenomena in Large Solid Propellant Boosters, with Application to the Ariane 5 Solid Rocket Motor (PhD thesis), Université Libre de Bruxelles, Belgium, 2000.

[18] J. Anthoine, J.-M. Buchlin, A. Hirschberg, Effect of nozzle cavity on resonance in large srm: theoretical modeling, J. Propul. Power 18 (2) (2002) 304-311.

[19] J. Anthoine, J.-M. Buchlin, J.-F. Guery, Effect of nozzle cavity on resonance in large srm: numerical simulations, J. Propul. Power 19 (3) (2003) 374-384.

[20] K.I. Matveev, F.E.C. Culick, A model for combustion instability involving vortex shedding, Combust. Sci. Technol. 175 (6) (2003) $1059-1083$.

[21] S. Gallier, M. Prevost, J. Hijlkema, Effects of cavity on thrust oscillations in subscale solid rocket motors, in: 45th AIAA/ASME/ASEE Joint Propulsion Conference \& Exhibit, AIAA, 2009. number 2009-5253.

[22] P. Durieu, G.C.J. Hofmans, G. Ajello, R.J.J. Boot, Y. Auregan, A. Hirschberg, Quasisteady aero-acoustic response of orifices, J. Acoust. Soc. Am. 110 (2) (2001) 1859-1872.

[23] B.E. Schmidt, Compressible Flow through Porous Media with Application to Injection, Technical report, California Institute of Technology, 2015.

[24] F.E. Marble, S.M. Candel, Acoustic disturbance from gas non-uniformities convected through a nozzle, J. Sound Vib. 55 (2) (November 1977 ) $225-243$.

[25] J. Anthoine, M.R. Lema, Passive control of pressure oscillations in solid rocket motors: cold-flow experiments, J. Propul. Power 25 (3) (2009) 792-800.

[26] L. Hirschberg, C. Schram, A. Hirschberg, Prediction of pulsations in a cold-gas scale-model of a solid rocket motor, in: 22nd AIAA/CEAS in Lyon, AIAA, 2016, pp. 1-22. number 2016-2826.

[27] D. Gilbarg, Handbuch der Physik: Strömungsmechanik III, Chapter Jets and Cavities, vol. IX, Springer-Verlag, Berlin, Germany, 1960.

[28] G.C.J. Hofmans, R.J.J. Boot, P.P.J.M. Durrieu, Y. Auregan, A. Hirschberg, Aeroacoustic response of a slit-shaped diaphragm in a pipe at low helmholtz number, 1: quasi-steady results, J. Sound Vib. 244 (1) (2001) 35-56.

[29] D. Yildiz, Influence of Radial Injected Flow on the Aeroacoustic Coupling in Solid Propellant Boosters, Technical report, VKI, Rhode-St-Genèse, Belgium, 2000.

[30] J.C. Bruggeman, A. Hirschberg, M.E.H. van Dongen, A.P.J. Wijnands, Self-sustained aero-acoustic pulsations in gas transport systems: experimental study of the influence of closed side branches, J. Sound Vib. 150 (3)(1991) 371-393.

[31] D. Tonon, A. Hirschberg, J. Golliard, S. Ziada, Aeroacoustics of pipe systems with closed branches, Int. J. Aeroacoustics 10 (2-3) (2011) 201-276.

[32] P.A. Nelson, N.A. Halliwell, P.E. Doak, Fluid dynamics of a flow excited resonance, part ii; flow acoustic interaction, J. Sound Vib. 91 (1983) $375-402$.

[33] D. Tonon, J.F.H. Willems, A. Hirschberg, Self-sustained oscillations in pipe systems with multiple deep side branches: prediction and reduction by detuning, J. Sound Vib. 330 (2011) 5894-5912.

[34] J. Golliard, S. Belfroid, A. Hirschberg, G. Nakiboglu, U-Rans model for the prediction of the acoustic sound power generated in a whistling corrugated pipe, in: ASME 2013 Pressure Vessels and Piping Conference, ASME, 2013, pp. 1-6. number PVP2013-97385.

[35] A. Michalke, On spatially growing disturbances in an inviscid shear layer, J. Fluid Mech. 23 (1965) 521-544.

[36] M.S. Howe, Acoustics of Fluid-structure Interactions, Cambridge University Press, Cambridge, UK, 1998.

[37] M.S. Howe, The dissipation of sound at an edge, J. Sound Vib. 70 (3) (1980) 407-411.

[38] P.A. Thompson, Compressible-Fluid Dynamics, MacGraw Hill, NY, USA, 1972.

[39] P. Henrici, Applied and Computational Complex Analysis, vol. I, Wiley-Interscience, NY, USA, 1974.

[40] L. Hirschberg, T. Schuller, C. Schram, J. Collinet, M. Yiao, A. Hirschberg, Interaction of a vortex with a contraction in a 2-dimensional channel: incompressible flow prediction of sound pulse, in: 23rd AIAA/CEAS Aeroacoustics Conference, AIAA, 2017. number 2017-3701.

[41] M.C.A.M. Peters, Aeroacoustic Sources in Internal Flows (PhD thesis), Technische Universiteit Eindhoven, Eindhoven, NL, 1993.

[42] S.J. Hulshoff, A. Hirschberg, G.C.J. Hofmans, Sound production of vortex nozzle interaction, J. Fluid Mech. 439 (2001) $335-352$.

[43] M.S. Howe, Hydrodynamics and Sound, Cambridge University Press, Cambridge, UK, 2007.

[44] R.R. Clements, An inviscid model for two-dimensional vortex shedding, J. Fluid Mech. 57 (1973) 321 -336. 